\title{
Percepções de portadores de diabetes sobre a doença: contribuições da Enfermagem
}

\author{
Perceptions of people with diabetes about the disease: nursing contributions
}

Percepciones de personas con diabetes acerca de la enfermedad: contribuciones de la Enfermería

\author{
Miriam de Oliveira Chaves', Mirian Rose Franco Teixeira', Sílvio Éder Dias da Silva" \\ ' Universidade Federal do Pará, Faculdade de Enfermagem (Graduanda). Belém-PA, Brasil. \\ "Universidade Federal do Pará, Faculdade de Enfermagem, Grupo de Estudos de História do \\ Conhecimento de Enfermagem e Saúde (GEHCES), Grupo de Pesquisa Educação, \\ Políticas e Tecnologia em Enfermagem da Amazônia (EPOTENA). Belém-PA, Brasil.
}

Submissão: 07-04-2011 Aprovação: 21-03-2013

\section{RESUMO}

Este estudo objetivou descrever a percepção dos usuários sobre a diabetes. A pesquisa foi descritiva e exploratória, com abordagem qualitativa; o cenário foi um hospital na cidade de Belém-PA, com participação de 32 sujeitos. Empregando-se a técnica de análise de conteúdo temática, emergiram quatro categorias: Controle do diabetes: a enfermagem na automonitorização da glicemia; O diabético e a enfermagem: uma interação para o autocuidado; Consulta de enfermagem ao diabético: a intervenção no processo saúde-doença; Diabetes e suas complicações: o medo repercutido na perda de funções. Observou-se que o paciente começa a se cuidar impulsionado pelo medo de perder sua saúde, obrigando-o ao autocuidado. O enfermeiro está diretamente ligado com o controle da diabetes, a partir do momento que realiza os cuidados e orientações da automonitorização da doença. Descritores: Diabetes; Educação em Saúde; Enfermagem.

\begin{abstract}
This study aimed to identify the perceptions of patients concerning diabetes. This is a descriptive exploratory study with a qualitative approach, which was conducted with 32 subjects, in a hospital in the city of Belem-PA, Brazil. The thematic content analysis was used, from which four categories emerged: Diabetes control: nursing in the self-monitoring of blood glucose; The diabetic individual and nursing: an interaction with self-care; Nursing consultations provided to diabetic individuals: an intervention in the health-disease continuum; Diabetes and its complications: fear of loosing functioning. It was observed that patients start self-care driven by the fear of losing their health, that is, fear forces the patient to practice self-care. Nurses are directly linked to diabetes control as they start providing care and guidance for patients who self-monitor their disease.
\end{abstract}

Key words: Diabetes; Health Education; Nursing.

\section{RESUMEN}

Este estudio tuve el objetivo de describir la percepción de los usuarios acerca de la diabetes. Realizó-se una investigación con enfoque descriptivo, exploratorio y cualitativo, en un hospital en la ciudad de Belém-Pará, Brasil, siendo entrevistados 32 sujetos. Fue empleada la técnica de análisis de contenido temático, siendo evidenciadas cuatro categorías: Control de la diabetes: la enfermería en el autocontrol de la glucemia; El diabético y la enfermería: una interacción para el autocuidado; Consulta de enfermería al paciente diabético: intervención en el proceso salud-enfermedad; Diabetes y sus complicaciones: el miedo refletado en la pérdida de funciones. Fue observado que el paciente comienza a cuidar impulsado por el miedo de perder su salud, y que el miedo hace que el paciente tenga el auto-cuidado de sí mismo. El enfermero está directamente conectado con el control de la diabetes, desde el momento en que realiza el cuidado y la orientación para la automonitorización de la enfermedad.

Palabras clave: Diabetes; Educación para la Salud; Enfermería.

\section{AUTOR CORRESPONDENTE Sílvio Éder Dias da Silva E-mail: silvioeder@ufpa.br}




\section{CONSIDERAÇÕES INICIAIS}

O objeto deste estudo diz respeito às percepções dos portadores de diabetes sobre a importância dos cuidados orientados pelos enfermeiros do Programa de Assistência ao Portador de Diabetes do HUJBB. O interesse pelo objeto de estudo surgiu após a oportunidade de conhecer o Programa de Diabetes do Hospital Universitário João de Barros Barreto (HUJBB) e observar o grande número de pacientes atendidos diariamente. Esse fato nos chamou a atenção para buscar avaliar se os pacientes atendidos conseguiam absorver as informações oferecidas pela equipe de enfermagem, visto que tais pacientes fazem uso do serviço destes profissionais permanentemente e o entendimento das informações prestadas influencia diretamente no sucesso do tratamento.

O diabete melito é um distúrbio crônico que afeta o metabolismo dos carboidratos, das gorduras e das proteínas. Um aspecto característico do diabete melito é a hiperglicemia, que constitui um reflexo da deterioração na utilização dos carboidratos (glicose) em virtude de uma resposta defeituosa ou deficiente à secreção de insulina ${ }^{(1)}$.

Em consequência das complicações crônicas, os diabéticos apresentam, em comparação à população não diabética, elevada morbidade e mortalidade duas a três vezes maiores, com redução na expectativa de vida ${ }^{(2)}$. Esta evolução indesejada pode ser amenizada com ações educativas voltadas para prevenção de agravos e manutenção da qualidade de vida.

Partindo desta ideia propomos o tema para analisar se as informações oferecidas aos diabéticos do Programa do HUJBB estão sendo assimiladas com sucesso, focalizando o compromisso do paciente com o tratamento e a ação da enfermagem na educação em saúde como forma de prevenção e promoção da saúde. Ressaltamos que, ao proporcionar orientações a este paciente, também promovemos prevenção, pois estimulamos um disseminador de informações; e que o aumento de diabéticos poderia ser amenizado ou parcialmente evitado pelo diagnóstico e tratamento precoce da doença e de suas complicações.

\section{OBJETIVO}

Descrever a percepção dos usuários a respeito do diabete melito e as implicações dessas percepções para as ações desenvolvidas pelos profissionais da enfermagem no Programa de Assistência ao Portador de Diabetes do HUJBB.

\section{METODOLOGIA}

Tratou-se de um estudo descritivo exploratório, realizado mediante abordagem qualitativa. Esta escolha ocorreu visto que foram os métodos que melhor atenderam os objetivos da pesquisa e que permitiram apreensão das opiniões e motivações expressas pelos participantes.

A pesquisa descritiva procura descobrir, com a precisão possível, a frequência com que um fenômeno ocorre, sua relação e conexão com os outros, sua natureza e características, correlacionando fatos ou fenômenos sem manipulá-lo ${ }^{(3)}$.
Alguns autores concordam com tal afirmativa, destacando que a pesquisa descritiva objetiva conhecer e interpretar a realidade, por meio da observação, descrição, classificação e interpretação de fenômenos, sem nela interferir para modificá-la ${ }^{(4)}$. As pesquisas exploratórias visam proporcionar uma visão geral de um determinado fato, do tipo aproximativo ${ }^{(5)}$.

A pesquisa qualitativa costuma ser direcionada ao longo de seu desenvolvimento, além disso, não busca enumerar e/ou medir os eventos estudados, seu foco de interesse é amplo e parte de uma perspectiva diferenciada da adotada pelo método quantitativo. Dela faz parte a obtenção de dados descritivos mediante contato direto e interativo do pesquisador com a situação objeto de estudo. Nas pesquisas qualitativas é frequente que o pesquisador procure entender os fenômenos segundo a perspectiva dos participantes da situação estudada e, a partir daí situe sua interpretação dos fenômenos estudados ${ }^{(6)}$.

A pesquisa foi desenvolvida no Hospital Universitário João de Barros Barreto (HUJBB), localizado no município de Belém do Pará. É uma instituição da Universidade Federal do Pará (UFPA) e tem como missão prestar assistência à saúde da população, por meio do Sistema Único de Saúde (SUS), como também atuar na área de Ensino e Pesquisa e na geração e sistematização de conhecimentos. É referência regional em Pneumologia, Infectologia e Endocrinologia e Diabetes, e Referência Nacional em AIDS. A residência médica em endocrinologia proporcionou o surgimento de pesquisas e com isso ocorreu à criação do Programa de Assistência ao Portador de Diabetes, onde os usuários são atendidos por uma equipe multiprofissional.

Participaram do estudo 32 indivíduos. Como critérios de inclusão, selecionamos usuários do Programa de Assistência ao Portador de Diabetes do HUJBB que fazem acompanhamento contínuo com a enfermagem e que concordaram em integrar este estudo.

Quanto ao aspecto ético, a proposta do estudo foi aprovada pelo Comitê de Ética em Pesquisa do Hospital Universitário João de Barros Barreto, sob o Protocolo n 1858/10 e foram observados os princípios éticos da pesquisa, obedecendo à Resolução no 196/96 (CNS), respeitando o rigor da pesquisa científica como a preservação do anonimato dos pacientes participantes da pesquisa.

Os dados foram coletados após a aprovação do Comitê de Ética em Pesquisa do Hospital Universitário João de Barros Barreto. A coleta foi realizada por meio da abordagem de autorrelato com as respostas dos participantes às perguntas colocadas pelo pesquisador, como em uma entrevista. Utilizou-se, ainda, um formulário contendo questões relativas aos objetivos que se pretendia alcançar ${ }^{(7)}$.

A pesquisa foi realizada com os pacientes no hospital, utilizando-se uma linguagem clara e compreensível, buscando informar esses pacientes a respeito da prevenção de agravos e importância da atenção prestada pela equipe de enfermagem.

Antes da entrevista tomamos conhecimento dos pacientes regularmente matriculados no programa e depois entrevistamos tais pacientes em suas consultas de rotina. Ressaltamos que todos receberam orientação a respeito do estudo e ficaram livres para aceitar ou recusar a sua participação na entrevista. 
Para proceder à análise do material coletado utilizamos a técnica de análise de conteúdo temática, que hoje é cada vez mais empregada para análise de material qualitativo obtido através de entrevistas de pesquisa( ${ }^{(8)}$.

A análise de conteúdo é um conjunto de técnicas de análise das comunicações visando obter, por procedimentos sistemáticos e objetivos de descrição do conteúdo das mensagens, a inferência de conhecimentos relativos às condições de produção/recepção destas mensagens ${ }^{(9)}$.

Para o tratamento dos dados foi utilizada a técnica da análise temática, que se baseia em operações de desmembramento do texto em unidades, ou seja, descobrir os diferentes núcleos de sentido que constituem a comunicação, e posteriormente, realizar o seu reagrupamento em classes ou categorias ${ }^{(9)}$.

$\mathrm{Na}$ exploração do material, tem-se o período mais duradouro: a etapa da codificação, na qual são feitos recortes em unidades de contexto e de registro; e a fase da categorização, no qual os requisitos para uma boa categoria são a exclusão mútua, homogeneidade, pertinência, objetividade e fidelidade e produtividade. Já a última fase, do tratamento e inferência à interpretação, permite que os conteúdos recolhidos se constituam em análises reflexivas, em observações individuais e gerais das entrevistas ${ }^{(9)}$.

Assim, dentro do discurso dos usuários do Programa de Diabetes foram observadas as seguintes temáticas: A. Controle do diabetes: a enfermagem na automonitorização da glicemia; B. O diabético e a enfermagem: uma interação para o autocuidado; C. Consulta de enfermagem ao diabético: a intervenção no processo saúde-doença; D. Diabetes e suas complicações: o medo repercutido na perda de funções.

\section{RESULTADOS E DISCUSSÃO}

\section{A. Controle do diabetes: a enfermagem na automonitori- zação da glicemia}

Nesta categoria observou-se que os depoentes necessitam fazer a automonitorização domiciliar, o que significa mudança na sua rotina diária e para que haja um bom andamento nesse processo, a enfermagem deve ensinar as praticas de automonitorização.

[...] Fui orientada pela enfermeira a furar o dedo, tirar a gota de sangue, colocar na fita e anotar o resultado (M1).

[...] Serve para fazer o acompanhamento e controlar o diabetes. Pegar informações para tratar (M16).

Observou-se que os sujeitos ao falarem sobre o controle da glicose, referiram terem sidos orientados pela enfermeira do programa a usarem o glicosímetro e ressaltaram que esta técnica é de suma importância para o controle da doença. Observamos com isso que os sujeitos conseguem compreender que a automonitorização da glicemia é importante para fazer o controle de sua doença. Avaliamos que esta prática de orientação ao usuário realizada pela enfermagem é extremamente importante, pois esta monitorização está intimamente relacionada com o bem estar do paciente em decorrência da diabetes ser uma patologia crônica que precisa ser constantemente monitorada para prevenir possíveis agravos.

Sabe-se que esta prática é essencial para dar direcionamento no tratamento, pois, de acordo com os valores obtidos, serão traçados novas condutas a respeito de medicações, alimentação e atividades físicas. É através desses valores, que o enfermeiro avalia se o tratamento está surtindo efeito e se o paciente aderiu a ele. Esta ação é bem visualizada pela enfermagem, pois são os profissionais que possuem um maior convívio com o paciente e servem de "ponte" para encaminhar, se necessário, o usuário para uma nova avaliação médica.

A monitorização da glicemia consiste em verificar seus valores com auxilio de um aparelho chamado glicosímetro, realizando uma punção digital. Sua finalidade é direcionar a terapêutica no sentido de obter e manter a glicemia o mais próximo possível da normalidade, em condições especiais ou na rotina diária, sempre com segurança. Os resultados da glicemia permitem compreender a interação entre medicação, atividade física e alimentação e a variabilidade glicêmica decorrente delas. Possibilitam identificar os sinais de hipo e hiperglicemia e prevenção das mesmas, diminuindo consideravelmente o risco de cetoacidose; conhecer a resposta glicemia alimentar e aos exercícios físicos ${ }^{(10)}$.

O monitoramento domiciliar da glicemia é um dos sete comportamentos para o autocuidado reconhecido pela AAED (American Association of Diabetes Educators) e é uma das mais importantes ações dentro do tratamento do $\mathrm{DM}^{(10)}$.

As recomendações para o controle domiciliar do diabético incluem automonitorização: da glicemia capilar, insulina, alterações dietéticas e realização de atividades físicas, a fim de manter os níveis glicêmicos. Assim, capacitar e dar suporte à pessoa diabética para o autocontrole domiciliar é uma responsabilidade conferida aos profissionais de saúde ${ }^{(11)}$.

\section{B. O diabético e a enfermagem: uma interação para o autocuidado}

Nesta categoria observou-se a relação da enfermagem em educar o paciente para que ele adquira conhecimentos que o ajudarão no seu autocuidado diário, isso implicar em uma mudança de hábitos na vida desses pacientes, tais mudanças leva tempo e acima de tudo o comprometimento do diabético com o seu tratamento, visando o seu bem estar. Dessa forma observamos também o comprometimento da Enfermagem em disseminar ensinamentos e orientações que levem o usuário do programa a ter o autocuidado consigo, promovendo com isso uma melhor qualidade de vida.

[...] Acho importante porque a enfermeira me orienta a controlar o diabetes e fala também as coisas que eu não posso comer (M2).

[...] Gosto muito de assistir as palestra da enfermeira. Ela me orienta sobre a insulina, uso do aparelho para verificar a glicose, o cuidado aos meus pés (M15).

O autocuidado é uma prática em que o paciente adquire informações que o ajudarão a cuidar de sua saúde, ele ganha 
com isso uma maior autonomia sobre a sua saúde, pois, ao adquirir conhecimento, ele irá desenvolver atividades diárias que trarão benefícios a si próprio. Com atitudes simples como verificar os pés, cuidados com os ferimentos, reeducação alimentar, o mesmo evitará possíveis complicações a sua saúde. O paciente passa a ter uma constante autovigilância sobre o seu corpo e sua saúde.

Os pacientes entrevistados mostraram-se conhecedores de sua patologia e dos agravos que a mesma pode trazer. A maioria pratica o autocuidado, tendo em vista o seu bem estar, e objetivam com isso evitar um possível agravo a sua saúde. A enfermagem tem se relacionado bem com os usuários do Programa, onde a principal meta alcançada é a adesão dos pacientes no seu tratamento diário o que ocorre na maior parte do tempo em seu domicilio. Dessa forma, os pacientes estão sendo bem orientados no seu autocuidado, sendo usuários conscientes, orientados e autônomos.

Sensibilizar os diabéticos para compreender essa necessidade de alterações pessoais no estilo de vida é papel fundamental dos profissionais envolvidos com o tratamento do diabetes. Adotar uma postura de decidir junto com o paciente, quais medidas são mais pertinentes e passíveis de execução, por meio de um processo colaborativo e não essencialmente prescritivo, encoraja-os a assumirem a responsabilidade de seu próprio controle e acredita-se, que somente assim as mudanças possam se concretizar ${ }^{(10)}$.

Na prática assistencial, deparamo-nos com o impacto que o DM tem sobre a saúde da população e com a dificuldade de adesão dos seus acometidos aos comportamentos preventivos. Nessa perspectiva, o enfermeiro, enquanto profissional de saúde engajado na assistência ao diabético, deve programar novas práticas de cuidado capazes de promover a saúde dos diabéticos, já que a adesão ao tratamento e o autocuidado são pontos frágeis da educação em saúde e que, portanto, merecem ser refletidos profundamente ${ }^{(12)}$.

Observa-se, dessa forma, o quanto é válido manter o diabético atento à importância da adesão ao tratamento por meio de educação continuada. Para tanto, precisa-se considerá-lo como um ser humano com suas crenças e como essas podem influenciar no modo como se cuida com o diabetes. Definimos crença como o resultado do domínio da experiência, constituindo-se de convicções não fundadas racionalmente e que modelam a conduta cotidiana. Fica claro então que, para orientarmos e estimularmos o autocuidado aos diabéticos, precisamos conhecer suas crenças e, além disso, fazê-lo conhecer como podem ser decisivas na forma como aprendem a se cuidar - componente cognitivo; decidem sobre como se cuidar - componente afetivo; e passam a se cuidar - componente comportamental ${ }^{(13)}$.

Utilizar os comportamentos de autocuidado na avaliação dos resultados educacionais a curto, médio e longo prazo deve ser o principal objetivo da enfermagem, para que seja monitorado o grau de conhecimento desses pacientes a cerca da prática desses autocuidados, pois sabemos que a diabete é uma doença crônica e precisa ser controlada continuamente. A enfermagem promove informações e ações educativas de suma importância para que o diabético se comprometa com sua saúde, e mais do que aprender ele irá colocar tudo em prática no seu dia a dia, observamos que a maioria dos entrevistados adquiriu bons hábitos após a consulta com o enfermeiro, dando mérito a esse profissional que o ensinou e orientou como fazer os seus cuidados diários.

\section{Consulta de enfermagem ao diabético: a intervenção no processo saúde-doença}

Nesta categoria observou-se a importância da assistência de enfermagem em ajudar o paciente a enfrentar a doença e adquirir um suporte profissional para ajudá-lo no melhoramento de sua qualidade de vida. Dessa forma observamos que o usuário absorveu que o trabalho da enfermagem é de extrema importância para dar seguimento no seu tratamento e proporcionar condições que favoreçam a boa qualidade do seu dia-a-dia.

[...] Antes de ter o Programa eu vivia jogado e com o Programa a gente encontra a equipe especializada (M8).

[...] Para a pessoa ser constantemente orientada, porque o diabetes é uma doença ingrata e temos que ser orientados (M6).

A enfermagem é uma profissão que se constrói na relação de confiança com o paciente. Esta profissão visualiza o paciente holisticamente, pois sabemos que o ser humano não deve ser visto apenas com um olhar, mas com visão biopsicossocial. Nas falas destacadas acima podemos perceber que os entrevistados expuseram claramente o seu bem estar em ser acompanhado pela equipe do Programa de Diabetes, e podemos também perceber que os usuários não enxergam apenas a sua doença, mas também o seu bem estar em ser acompanhado e tratado. Podemos visualizar tal fato na fala do entrevistado M8 que afirmou viver jogado e agora tem uma equipe especializada. Isto nos reporta que o usuário do programa possui uma percepção de amparo e cuidado por sua saúde e também por sua doença como relata os demais entrevistados ao referirem acompanhamento para controle e tratamento.

Deste modo, podemos perceber que o diabético do programa encontra-se familiarizado com a enfermagem e que tal situação reflete no bem estar físico e emocional, que muito ajuda em seu tratamento visto que a doença crônica faz a pessoa ficar dependente do tratamento.

O conceito de promoção da saúde aponta para uma visão holística do processo saúde-doença e da forma de intervir nesse processo. Nesse sentido, a abordagem multiprofissional do indivíduo diabético remete a essa visão holística, reconhecendo a complexidade do seu sistema psíquico e somático que, por isso, necessita de informações complementares dos profissionais de saúde sobre o controle, a prevenção e as complicações da sua doença ${ }^{(14)}$.

Em 2003, foi implementada a consulta de enfermagem ao paciente diabético, numa abordagem psicossocial, levando em consideração uma atuação voltada à ampliação, tentando alçá-la à abordagem da pessoa, suas interações consigo, com o outro, com o contexto, enfim, com tudo que está fora, porém não separado dela mesma, incluindo as expressões de saúde-doença ${ }^{(15)}$.

A abordagem psicossocial é bastante válida, principalmente para os profissionais que querem promover a autonomia 
das pessoas e que acreditam que ainda é possível melhorar a qualidade de vida das pessoas, mesmo quando essas já têm uma doença crônica(15).

Ao analisar a fala do entrevistado M6 observamos que ele define o diabete como uma doença ingrata. Com isso podemos perceber, que este paciente necessita de uma orientação um pouco mais voltada para as suas particularidades, pois sabemos que a percepção de cada pessoa a respeito de sua doença é muito diversificada.

Há necessidade de sabermos mais sobre a experiência individual do diabético, o ambiente físico e social, a influência da cultura na definição da doença e as atitudes para com ela ${ }^{(16)}$.

Quando se faz referência ao processo educativo para o controle do diabetes, fala-se da importância de se adotar estratégias, cujas ações devem ter caráter participativo tanto do indivíduo quanto de sua família. Sabe-se que as ações educativas influenciam o estilo de vida, melhoram a relação profissional-indivíduo e os ambientes social e físico. Além disso, a educação em saúde, como uma prática social, baseada no diálogo, ou seja, na troca de saberes, favorece a compreensão dessa relação no processo saúde-doença e, respectivamente, o intercâmbio entre o saber científico e o popular ${ }^{(17)}$.

A abordagem psicossocial é bastante valida, principalmente para os profissionais que querem promover a autonomia das pessoas e que acreditam que ainda é possível melhorar a qualidade de vida das pessoas, mesmo quando estas já têm uma doença crônica. Deste modo ao se ampliar a abordagem da pessoa com diabetes leva em consideração, não somente os aspectos clínicos, tão fortemente estudados, mas também os aspectos psicossociais que constituem muitas vezes, determinante do processo saúde doença ${ }^{(15)}$.

Sendo assim, ressalta-se a importância do enfermeiro visualizar holisticamente estes pacientes, para que as consultas de enfermagem possam dirigir um tratamento que vise amenizar os desconfortos da doença e prevenir possíveis agravos o que gera promoção da saúde.

\section{Diabetes e suas complicações: o medo repercutido na perda de funções}

Observa-se nesse tópico o medo que o paciente diabético tem de perder alguma função, ou mesmo de perder uma parte do seu corpo, por conta das complicações do diabete. O paciente começa a se cuidar impulsionado pelo medo de perder sua saúde e até mesmo ficar mutilado, dessa forma o medo obriga o paciente ao autocuidado.

[...] Problema no rim, cegueira, feridas que dão na perna, perda de dedo, pé e perna (M21).

\section{[...] Posso ficar cega, perder um braço ou uma perna, posso} ficar doente (M2).

O paciente com diabetes tem uma percepção que a doença agora faz parte do seu mundo e necessita conhecer os controles necessários para manter uma boa qualidade de vida, mas alguns relutam em abandonar hábitos adquiridos num passado sem a doença. O que o motiva à mudança desses hábitos é o medo das complicações que o diabete pode trazer para sua vida. Eles têm medo de ficar limitados, dependentes de alguém ou de algo; assim sendo, algumas pessoas expressaram claramente seu temor por uma amputação, por uma cegueira, pela possibilidade de perder sua capacidade de ir e vir, de cuidar de si mesma. A verbalização da perda não apenas como impossibilidade inerente à condição humana, mas potencializada pelas possíveis complicações da Diabetes está presente em algumas falas.

\section{[...] A enfermeira trata a gente bem, com carinho. Ela orien- ta a gente e não briga com a gente (M23).}

No que tange à enfermagem, o enfermeiro deve ver o paciente de uma forma holística não apenas como uma pessoa que está doente, mas atentar como essa pessoa está reagindo e enfrentando essa patologia crônica com que terá que conviver no seu cotidiano. A enfermagem deve avaliar e dar suporte emocional a esse usuário, visto que para êxito no tratamento o paciente precisa estar com a diabete controlada, necessita se sentir saudável e com uma boa qualidade de vida, longe de medos, mas esclarecido sobre sua patologia e como o mesmo pode ajudar no seu tratamento.

O medo nada mais é que a falta de confiança que o tratamento não tenha efeito sobre sua doença, visto que se os usuários do Programa ao cuidarem muito bem de sua saúde, não deverão temer essas complicações, pois os mesmos estarão prevenindo-as. Esse medo é consequência do "sentir-se doente" e ter que conviver com uma doença crônica para o resto de sua vida. Faz-se extremamente necessário a atuação da enfermagem em tratar esse medo do paciente criando vínculos de amizade com esse usuário, onde o paciente veja o enfermeiro não como alguém que o amedronta e o obriga a se cuidar, mas como um facilitador de conhecimento que promoverá o autocuidado, ele está ali para esclarecer dúvidas, para ajudar e não amedrontar.

O enfermeiro deve atentar para o modo como esse paciente expressa a situação existencial concreta do ser doente, vinculada à historicidade própria da doença de cada um, visto serem pessoas de hábitos e costumes diferentes. No processo ensino-aprendizagem há obstáculos, tanto cognitivos como emocionais, que precisam ser compreendidos e trabalhados pela enfermagem.

O grande número de investigações relacionadas aos doentes de diabete tem permitido a continuidade dos programas de cuidado à doença. Entretanto, os resultados não têm sido favoráveis devido às complicações surgidas. Assim, é importante dar continuidade aos estudos de forma a atingir melhores condições de vida para estes doentes, considerando que a enfermidade se relaciona a sentimentos negativos, como choque, revolta e tristeza ${ }^{(18)}$.

A medicina sozinha não consegue dar conta de ajudar no controle da doença para que não haja complicações, pois além de exames e medicamentos, é necessário dieta, exercícios físicos e um equilíbrio emocional, visto que estes aspectos influem diretamente sobre a doença ${ }^{(19)}$.

A doença psicossomática é qualquer alteração somática (física) decorrente de sofrimentos psíquicos, diferentemente da 
somatopsíquica que é qualquer alteração psíquica decorrente de sofrimento físico, por exemplo, os efeitos psíquicos sofridos pelo indivíduo que possui uma enfermidade crônica ou uma debilidade física. Sendo assim o diabetes mellitus pode ser considerado tanto uma doença psicossomática quanto somatopsíquica ${ }^{(20)}$.

A doença psicossomática surge em decorrência do modo como o indivíduo vivencia as emoções. As emoções (medo, ira, amor) são situações novas frente às quais o organismo se desequilibra e se prepara para descarregá-las através dos músculos voluntários do corpo. Porém, muitas vezes, as emoções não são descarregadas, expressas, talvez pela imposição da sociedade para reprimi-las. Quando as emoções não são expressas através de nossos músculos voluntários, elas descarregam-se em nossos músculos involuntários, como o estômago, intestino, coração e vasos sanguíneos, podendo desencadear a doença psicossomática(20).

O diabético transporta o emocional (medo de sofrer) para o soma (não permitir a entrada do açúcar, simbolicamente do amor). Isso também pode ser observado na alteração da glicemia. Quando o diabético vive uma tensão emocional há um aumento de glicemia, assim quando o diabético fica tenso permite menos ainda que o açúcar (doce) entre na célula. O emocional é constituído por aspectos mais profundos internamente e inconscientes, que podem impedir um bom controle da doença se esta não for internamente aceita ${ }^{(21)}$. Acredita que o diabetes será enfrentado diferentemente por cada indivíduo, pois dependerá da estrutura psíquica ou organização mental de cada um ${ }^{(22)}$.

\section{CONSIDERAÇÕES FINAIS}

A pesquisa buscou conhecer e descrever a percepção dos usuários do Programa de Diabetes do HUJBB a respeito da significância do profissional enfermeiro dentro do Programa, identificando-se que, para tais usuários, a enfermagem contribui positivamente para a manutenção de sua saúde e da sua qualidade de vida, ao ensiná-lo para o autocuidado.

Dentro das análises realizadas, observamos que o enfermeiro está diretamente ligado com o controle do diabetes, a partir do momento que realiza os cuidados e orientações da automonitorização da doença. Isto mostra que cabe à enfermagem ensinar os pacientes a praticarem esta monitorização, pois encontramos também o enfermeiro interagindo com os usuários e facilitando assim o seu autocuidado, que em se tratando de pacientes crônicos deve ser focado continuamente.

Observamos também que a consulta de enfermagem possui uma grande importância para os usuários, pois estes passaram a conviver melhor com a doença a partir do momento que criaram vínculos com o enfermeiro que continuamente promove ações que melhoram a qualidade de vida em função de trabalharem no processo saúde-doença. Logo, tal vínculo foi explicito quando observamos o medo dos pacientes em relação às perdas ocasionadas por agravos, o que nos reportou que estes pacientes conseguiram absorver as possíveis consequências que podem ocorrer caso eles não assumam as responsabilidades do seu tratamento.

O enfermeiro é o profissional que serve como uma seta planejadora de ações, pois atende o usuário de maneira individualizada, avaliando-o e encaminhando-o a outros profissionais quando necessário. Compreendemos que o enfermeiro é visto como um amigo e facilitador no autocuidado dos pacientes, pois a maioria dos entrevistados colocou que as "orientações e ensinamentos" feitos pelo enfermeiro foram fundamentais para o aprendizado na realização do cuidado de si.

Do total de entrevistados, $97 \%$ consideram a consulta com o enfermeiro importante e de grande valia, pois, por meio da consulta, tiveram uma melhora significativa nas suas vidas; $100 \%$ referiu que o papel do enfermeiro é ensinar e orientar no seu autocuidado. Entre as ações de autocuidado estão a monitorização do diabetes, com verificação periódica da glicemia, uso de insulina, cuidados com os pés, cuidados com ferimentos, a reeducação alimentar, os agravos e complicações ocasionados pela diabetes.

Entre o déficit de conhecimento, está a importância de esse usuário ir ao oftalmologista regularmente, visto que a diabetes pode levar a cegueira. Ressalte-se que 50\% dos entrevistados relataram não terem sido orientados sobre a importância de ir ao oftalmologista; os outros $50 \%$ foram orientados sobre a importância e vão regulamente ao oftalmologista. Dessa forma fica um tema a ser trabalhando mais pela equipe de enfermagem do programa sobre os cuidados que os pacientes com diabetes devem ter com sua visão. Cerca de $60 \%$ não foram orientados sobre a importância de usar calçados adequados, e 37\% informarão terem sido orientados e fazem o uso de sapados adequados.

A maioria dos usuários está absorvendo e realizando as orientações que são repassadas pelo profissional enfermeiro, alguns relatam que sentem saudades das palestras que anteriormente eram feitas pela enfermeira do Programa, hoje os cuidados e orientações são individualizados feitos durante as Consultas da enfermeira. Uma vez que é de extrema importância à educação em saúde feita por estes profissionais fica mais uma sugestão sobre a volta das ações educativas em grupos, onde há uma troca de saber de um usuário com outro.

Os dados obtidos nessa investigação mostraram que o processo educativo oferecido pelo Programa de Diabetes do HUJBB, durante a Consulta de Enfermagem teve repercussão na dinâmica do usuário, ampliando os seus conhecimentos em relação ao tratamento e controle da diabetes. As falas obtidas possibilitaram compreender que o paciente constituiu um elemento que expande o conhecimento acerca da diabetes no seu cotidiano e este passou a exercer mais adequadamente seu papel no seu autocuidado. A ampliação do aprendizado pode favorecer a aquisição de hábitos saudáveis na vida desse diabético. Desse modo, compreendemos que a educação em diabetes deve estar centrada na significância que o enfermeiro exerce na equipe multidisciplinar dentro do programa e na vida desses usuários. Quando o paciente encontra essa rede de apoio na Enfermagem, há uma maior efetividade no processo educativo.

Dentro dos objetivos propostos concluímos que os usuários do Programa de Assistência aos Portadores de Diabetes do HUJBB possuem uma boa percepção a respeito dos cuidados repassados pela equipe de enfermagem, isto nos reforça a importância do trabalho destes enfermeiros como educadores da saúde com intuito de promover uma melhor qualidade de vida para esses usuários. 


\section{REFERÊNCIAS}

1. Cotran RS, Kumar V, Robbins ST. Robbins patologia estrutural e funcional. 4.ed. Rio de Janeiro: Guanabara Koogan; 1991.

2. Forti A, Loureiro R, Gusmão A, Teixeira L. Diabetes Mellitus - Classificação e Diagnóstico. In: VILAR L. Endocrinologia clínica. 3.ed. Rio de Janeiro: Guanabara Koogan; 2006. p.539-548.

3. Certo AL, Bervian PA. Metodologia científica. 4. Ed. São Paulo: Makron Books; 1996. p.90.

4. Vieira VA. As tipologias, variações e características da pesquisa de marketing. Revista FAE 2002;5(1):61-70.

5. Gil AC. Como elaborar projetos de pesquisa. São Paulo: Atlas; 2002.

6. Godoy AS. Introdução a pesquisa qualitativa e suas possibilidades. Rev Adm Emp 1995;35(2).

7. Polit DF, Beck CT, Hungler BP. Avaliação dos métodos de coleta de dados. In Polit DF, Beck CT, Hungler BP. Fundamentos de pesquisa em enfermagem: métodos, avaliação e utilização. 5.ed. Porto Alegre: Artmed; 2001. p.247-284.

8. Machado MNM. Entrevista de pesquisa: a interação entrevistador / entrevistado. Belo Horizonte. Tese [Doutorado em Enfermagem] -Universidade de Belo Horizonte; 1991.

9. Bardin L. Análise de conteúdo. Lisboa: Edições 70; 2002.

10. Grossi SAA, Pascali PM, organizadora. Cuidados de enfermagem em diabetes mellitus. Departamento de enfermagem da Sociedade Brasileira de Diabetes. Manual de Enfermagem. São Paulo; 2009.

11. Zanetti ML. O diabetes mellitus tipo 1 em crianças e adolescentes: um desafio para as mães e profissionais da saúde. Ribeirão Preto. Tese [Doutorado em Enfermagem] - Escola de Enfermagem de Ribeirão Preto, Universidade de São Paulo; 1996.

12. Ataide MB. C. Vivência grupal: estratégia de engajamento no autocuidado e diabetes. Fortaleza. Tese[Doutorado em Enfermagem] -Universidade Federal do Ceará; 2004.
13. Xavier TDF, Bittar AB, Barroso DCDA. Crenças no autocuidado em diabetes - implicações para a prática. Texto \& Contexto Enferm 2009;18(1):124-130.

14. Fernandez LAL, Regules JMA. 1994. Promoción de Salud: Un Enfoque en Salud Pública. Documentos Técnicos. Granada: Escuela Andaluza de Salud Pública. In: Torres HC, Hortale VA, Schall V. A experiência de jogos em grupos operativos na educação em saúde para diabéticos. Cad Saúde Pública 2003;19(4).

15. Rodrigues FPC. A consulta de enfermagem centrada na promoção à saúde do adulto portador de diabetes mellitus: uma abordagem psicossocial. São Paulo. Dissertação [Mestrado em Enfermagem] -Escola de Enfermagem da USP; 2003.

16. Restrepo HM. Promoción de la Salud: Como Construir Vida Saludable. Bogotá: Editora Médica Internacional; 2001. In: Torres HC, Hortale VA, Schall V. A experiência de jogos em grupos operativos na educação em saúde para diabéticos. Cad Saúde Pública 2003;19(4).

17. Briceño-Leon, R. Siete tesis sobre la educación sanitaria para la participación comunitária. Cad Saúde Pública 1996:12:7-30. In: Torres HC, Hortale VA, Schall VA. A experiência de jogos em grupos operativos na educação em saúde para diabéticos. Cad Saúde Pública 2003;19(4).

18. Oviedo AD, Boemer MR. A pessoa com diabete: do enfoque terapêutico ao existencial. Rev Esc Enferm USP 2009;43(4).

19. GEED- Grupo de Estudos em Endocrinologia \& Diabetes. Proposta de um estudo multicêntrico com diabéticos em uso de insulina. Endocrinologia \& Diabetes Clínica e Experimental (Curitiba) 2001;1(5): 15-18.

20. Silva MAD. Quem ama não adoece. São Paulo: Best Seller;1994.

21. Marcelino DB, Carvalho MDB. Reflexões sobre o Diabetes Tipo 1 e sua Relação com o Emocional. Psicol Reflex Crít 2005;18(1):72-77.

22. Debray R. O equilíbrio psicossomático: Um estudo sobre diabéticos. São Paulo: Casa do Psicólogo; 1995. 